UCRL-ID-121339

PREPRINT

\title{
Derivation of Preliminary Specifications for Transmitted Wavefront and Surface Roughness for Large Optics Used in Inertial Confinement Fusion
}

Dave Aikens, Lawrence Livermore National Laboratory

Andre Roussel and Michael Bray, CEA-DAM

Centre d'etude de Limeil-Valenton (CEL-V), DLPP/EME/TL

94195 Villeneuve St Georges Cedex

June 27,1995

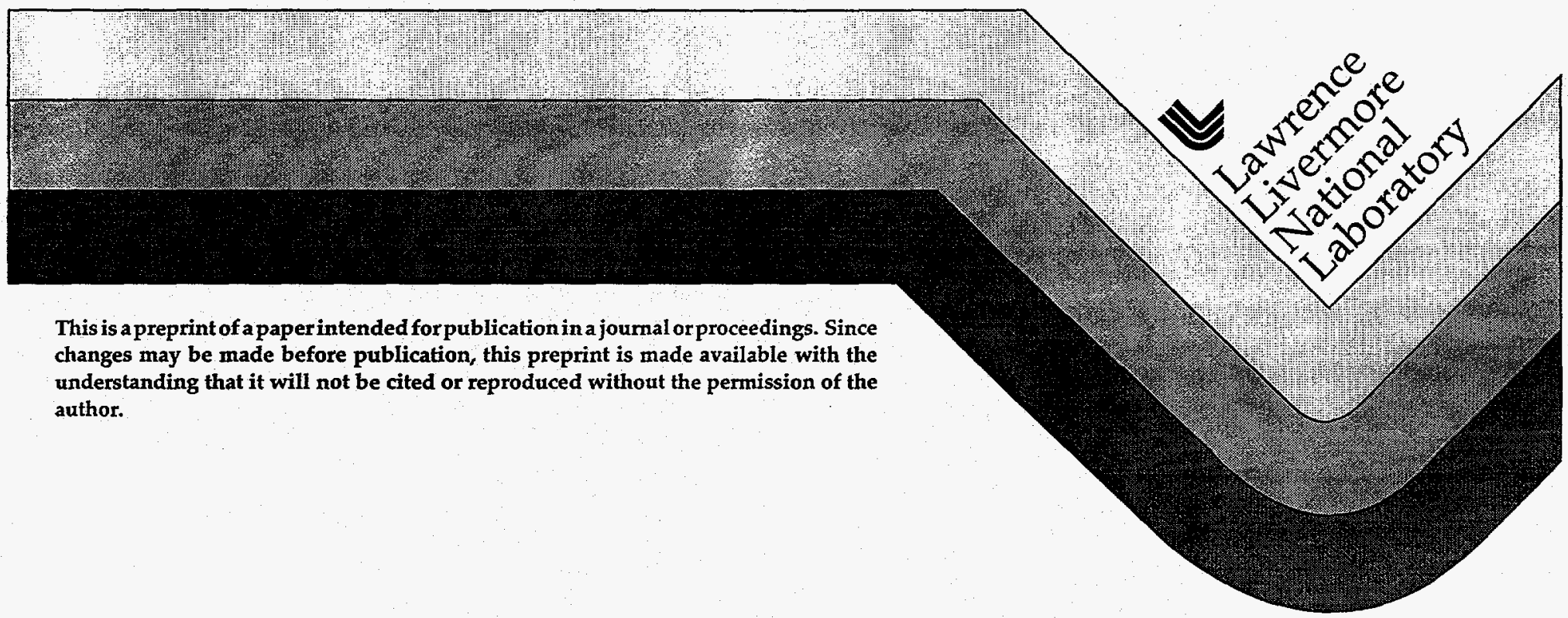




\section{DISCIAIMER}

This document was prepared as an account of work sponsored by an agency of the United States Government. Neither the United States Government nor the University of California nor any of their employees, makes any warranty, express or implied, or assumes any legal liability or responsibility for the accuracy, completeness, or usefulness of any information, apparatus, product, or process disclosed, or represents that its use would not infringe privately owned rights. Reference herein to any specific commercial product, process, or service by trade name, trademark, manufacturer, or otherwise, does not necessarily constitute or imply its endorsement, recommendation, or favoring by the United States Government or the University of California. The views and opinions of authors expressed herein do not necessarily state or reflect those of the United States Government or the University of California, and shall not be used for advertising or product endorsement purposes. 


\section{DISCLAIMER}

Portions of this document may be illegible in electronic image products. Images are produced from the best available original document. 


\title{
Derivation of preliminary specifications for transmitted wavefront and surface roughness for large optics
} used in Inertial Confinement Fusion

Dave Aikens

\author{
Lawrence Livermore National Laboratory \\ P.O. Box 808, L-487, Livermore, CA 94551 \\ André Roussel, Michael Bray \\ CEA-DAM - Centre d'étude de Limeil-Valenton (CEL-V), \\ DLPP/EME/TL, 94195 Villeneuve St Georges Cedex
}

\begin{abstract}
In preparation for beginning the design of the Nation Ignition Facility (NIF) in the United States and the Laser Méga-Joule (LMJ) in France, we are in the process of deriving new specifications for the large optics required for these facilities. They are currently being evaluated through modeling and experimentation. These specifications will be ready for general release by the end of the year.
\end{abstract}

Traditionally, specifications for transmitted wavefront and surface roughness of large ICF optics have been based on parameters which were easily measured during the early 1980's, such as peak-to-valley wavefront error (PV) and root-mean-square (RMS) surface roughness, as well as wavefront gradients in terms of waves per $\mathrm{cm}$. While this was convenient from a fabrication perspective, since the specifications could be easily interpreted by fabricators in terms which were understood and conventionally measurable, it did not accurately reflect the requirements of the laser system. In some cases, optics which were not adequate for a given application which was particularly sensitive to periodic errors, were fabricated acceptably in terms of the optics specifications. For the NIF and LMJ laser systems, we have availed ourselves of advances in metrology and interferometry and an enhanced understanding of laser system performance to derive specifications which are based on power spectral densities (PSD's.) Such requirements can more accurately reflect the requirements of the laser system for minimizing the amplitude of mid-and high-spatial frequency surface and transmitted wavefront errors, while not over constraining the fabrication in terms of low spatial frequencies, such as residual coma or astigmatism, which are typically of a very large amplitude compared to periodic errors.

In order to study the effect of changes in individual component tolerances, it is most useful to have a model capable of simulating real behavior. The basis of this model is discussed in this paper, outlining the general approach to the "theoretical" study of ICF optics specifications, and an indication of the type of specification to be expected will be shown, based upon existing ICF laser optics. The problem of specifying optics for high energy lasers is more difficult than for "classical" optical systems for-many" reasons, which is discussed as well.

Keywords: ICF, high energy lasers, optical components, specifications, power spectral density, PSD.

\section{SPECIFICATIONS IN GENERAL}

Specifying optics for ICF lasers, or any optical system, is the task of reducing the cost of optics while maintaining quality. In order to do this, it is necessary to have knowledge of two quantities: the cost of optical components versus quality, which depends on the capability of suppliers, and the actual laser requirements.

Also, it is essential that the specifications have the two following qualities in order to be applicable:

a. Simplicity: they must be easy to understand and to apply in the workshop.

b. Accuracy: they must reflect the desired quality exactly. 
These two requirements are, of course, contradictory. The usual specification for ICF optics (NOVA and Phebus in the 1980 's, etc.) was to specify distortion of the transmitted wavefront of the component in terms of the Peak-to-Valley (in waves) and of the gradient (in waves-per-cm) The values for these specifications were selected based on 'rules of thumb' and experience. Given the metrology capabilities of the time, this approach was the most logical. Today, however, with advances in metrology and changes in finishing processes, it is both possible and necessary to apply more accurate specifications which can be remain relatively simple through the application of existing metrology tools and commercially available software.

\section{DIFFERENCE BETWEEN ICF OPTICS AND CLASSICAL OPTICS}

The main differences between the specifying of ICF optics and that of classical optics are the following:

a. ICF components are large, typically 400 to $800 \mathrm{~mm}$. Therefore, the scale length of shape errors covers almost six decades (1000 to $0.001 \mathrm{~mm}$ ).

b. ICF lasers are long systems, typically tens to hundreds of meters. This, coupled with (a.) above, results in a wide range of Fresnel lengths which can fall within the system, equivalent to feature sizes of $10 \mathrm{~mm}$ to $0.001 \mathrm{~mm}$.

c. ICF lasers are created using non-linear effects, which can result in power related amplification of spatial inhomogeneity. These effects can be quite dramatic. ${ }^{1}$

d. ICF Lasers contain a wide variety of components, such as: silica aspheric off-axis focusing lenses, standard-polished laser glass slabs, fly-cut KDP frequency converters, etc. Thus, the expected cost versus quality trade-off is different for different components.

The various physical and optical phenomena encountered during the specifying of ICF optics depend on such values as feature scale and/or Fresnel length. Therefore, their behavior will be of a different nature in each scale range.

\section{ICF AMPLIFIER SECTION - A DESCRIPTION}

Figure 1 shows a simplified diagram of a typical amplifier section. The three basic components of the section are:

a. Input filtering, including a collimating lens

b. Laser amplifier slabs; there are between three and ten per section, tilted at Brewster'sangle.

c. Output filtering, including focusing lens.

The purpose of the filtering sections is to remove those higher frequencies that might experience high gain. The size of the holes is calculated so that the main beam may pass through without coming too near their edge, which would be fatal to the beam propagation. In the particular case of NIF and Méga-Joule, this size is equivalent to a ripple size of about $10 \mathrm{~mm}$.

The propagation mechanism is the following: An incoming beam focuses on the input filtering hole, is cleaned of its higher spatial frequencies which diffract outside the clear aperture. It then experiences energy amplification as it goes through the slabs. Irregularities in the wavefront are unintentionally amplified by non-linear processes, in a manner explained below. At the output filtering, the higher frequencies will not pass the hole, as they diffract outside its clear aperture, but the lower frequencies will get through and will go on building up through the entire laser chain. 


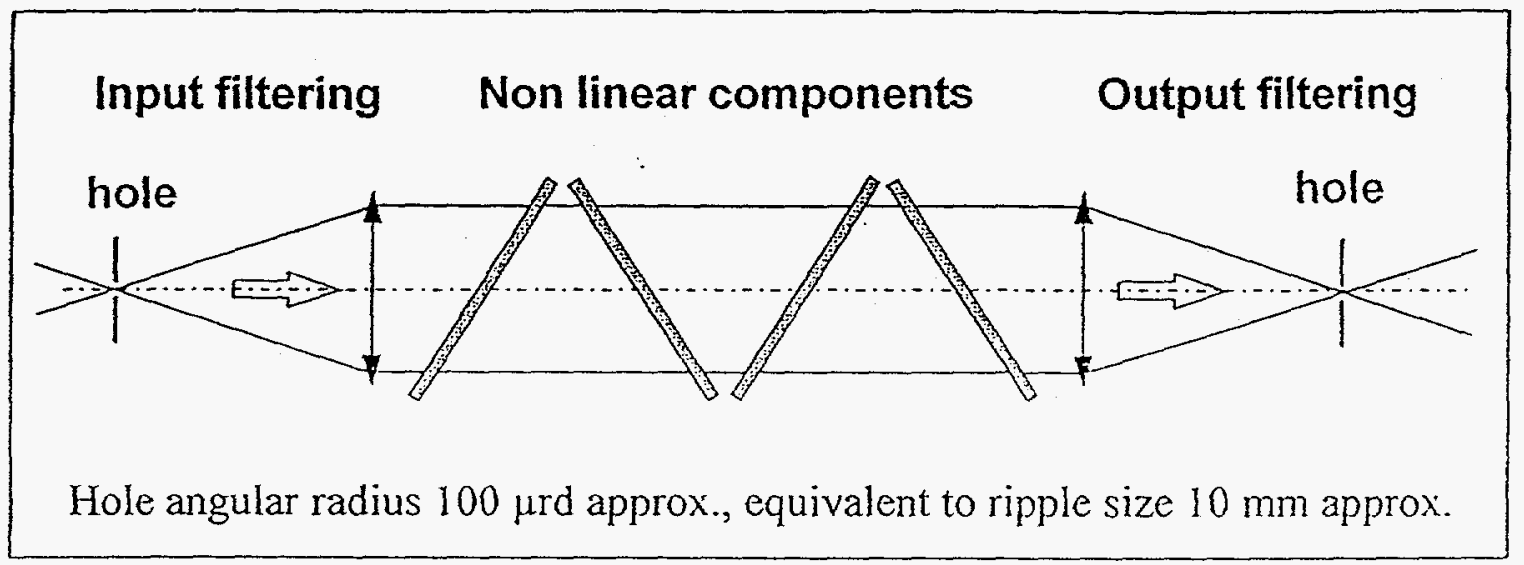

Fig. 1. Simplified diagram of an amplifier section, including non-linear material.

$$
E(x, y, z)=E_{0}\{1+a(z) \cdot e(x, y)\} \quad a(z)=u(z)+i \cdot v(z)
$$

$\left.\begin{array}{l}u: \text { real part : mode amplitude } \\ v: \text { imaginary part : mode phase }\end{array}\right\}$ Mode Power: $u^{2}+v^{2}$

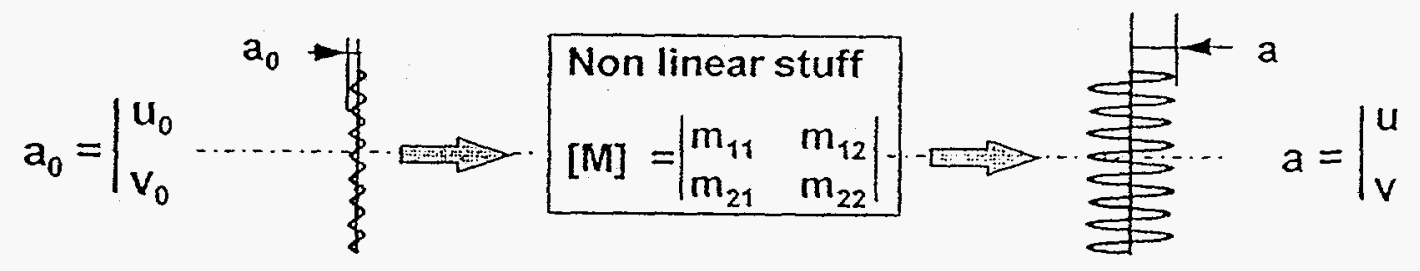

- MODE AVERAGE POWER over spatial frequencies relates to ENERGY LOSS.

- MODE PEAK AMPLITUDE relates to OPTICS DESTRUCTION.

Fig. 2. Summary of the basic model used in this paper. 
Suppose there is a periodic error in the phase of the laser, caused by an error in the transmitted wavefront of, for instance, the collimating lens. That phase error will be converted to amplitude error through normal diffraction and amplified as discussed above. The reader unfamiliar with non-linear processes might ask to what extent these processes can be significant. An idea of their importance can be illustrated by the following occurrence: during operation on Beamlet (a test laser for National Ignition Facility), in which optical components are specified as in NOVA, unacceptably large modulation of intensity was present after two passes, and could not be accounted for. The problem was later traced to one laser slab, which showed ripple of spatial period $12 \mathrm{~mm}$, and an exceedingly low amplitude, around $\lambda / 100$ Peak-to-Valley (P-V). When the offending optic was removed, the amplitude modulation did as well. Clearly, non-linear processes are important.

\section{SIMPLIFIED THEORY OF NON-LINEAR RIPPLE GROWTH}

\subsection{Basics}

First, we will write the relation between beam power and index of refraction:

$$
\mathrm{n}=\mathrm{n}_{\mathrm{O}}+\gamma \cdot \mathrm{p}
$$

where $n_{0}$ is the index of refraction of the material at zero beam power, $\gamma$ is the non-linear parameter, and $P$ the power density of the beam. It is customary to express $P$ in Watts $/ \mathrm{cm}^{2}$, therefore $\gamma$ is in $\mathrm{cm} 2 / W$ att.

Classical numerical values for laser glass are: $n_{0}=1.5 ; \gamma=2 \cdot 10^{-16} \mathrm{~cm}^{2} / \mathrm{W}$. With $P=5 \mathrm{GW} / \mathrm{cm}^{2}$ (approximately the value at the last slab of NIF and Méga-Joule), the index variation is:

$$
\mathrm{dn}=\gamma \cdot \mathrm{p}=1 \cdot 10^{-6}
$$

Propagating through a thickness of laser glass $t$, in a beam of wavelength $\lambda$, produces a wavefront phase variation equal to:

$$
d \phi=(2 \pi / \lambda) \cdot t \cdot d n
$$

With values of $t=40 \mathrm{~mm}, \lambda=1053 \mathrm{~nm}$, we have:

$$
\mathrm{d} \phi=0.24 \text { (radians) }
$$

This value is quite large: for ten slabs, a typical value for $d \phi$ will be 2.0 (the power density increases in one amplifier section to its maximum value computed above, so $\mathrm{d} \phi$ does the same). This means the phase retardation of a plane wavefront will be 2 radians, or about $\lambda / 3$.

A final important parameter which will turn up later is the critical period, $p_{c}$, defined as:

$$
\begin{gathered}
\left(2 \pi / \mathrm{p}_{\mathrm{c}}\right)=2 \cdot \mathrm{k} \cdot\left(\mathrm{dn} / \mathrm{n}_{\mathrm{o}}\right)^{1 / 2} \\
\text { or: } \mathrm{p}_{\mathrm{c}}=(1 / 2) \cdot\left(\lambda / \mathrm{n}_{\mathrm{O}}\right) \cdot\left(\mathrm{dn} / \mathrm{n}_{\mathrm{o}}\right)^{-1 / 2}
\end{gathered}
$$

where $\mathrm{k}=(2 \pi) \cdot \mathrm{n}_{0} / \lambda$, or the wave number.

With the numerical values given previously, we find:

$$
\mathrm{p}_{\mathrm{c}}=0.43 \mathrm{~mm} .
$$

\subsection{Small Ripple Amplification (SRA)}

Ripple amplification mechanism is the following: a ripple ("parent" ripple) launches near-field waves, which induce a periodic power variation of the main beam, which in turn impresses a periodic index 
variation in non-linear materials. Effectively, a hologram has been created. This hologram diffracts the main beam which, being powerful, gives rise to a "child" ripple, of same periodicity as the initial ripple, but with an amplitude which can be anywhere from zero up to tens of times that of the "parent" ripple, depending mainly on the spatial ripple size.

This process is "self-amplifying", and it is therefore not surprising to find that the maximum ripple gain depends exponentially on $d \phi$. Because of its importance, it is given a special symbol, B.

$$
\mathrm{B}=\Sigma \mathrm{k} \cdot \mathrm{t} \cdot \mathrm{dn} \text { (sum over beam path) }
$$

Figure 2 shows a summary of the parameters of significance in non-linear amplification. We will not attempt to demonstrate the derivation of the Small Ripple Amplification matrix formulation, as it is well documented elsewhere. ${ }^{2}$ Rather, we will derive from it the various equations relating directly to the subject of optics specifications.

First, the notion of ripple amplitude must be clarified. A plane wave can be perturbed in two "orthogonal" ways : in amplitude and in phase. The former affects the power density in the beam, the latter affects the shape of the wavefront. However, both have the capability of diffracting light out of the main beam. If we expand the equation for the amplitude of a compound perturbation of a wave, we have

$$
A=A_{0} \cdot(1+u) \cdot e^{i v}
$$

where $A$ is the resulting amplitude, $A 0$ the unperturbed amplitude, $u$ the relative amplitude variation, and $v$ the phase variation. A first order expansion in the variables yields (assuming $v$ is small):

$$
A=A_{0} \cdot(I+u+i v)=A_{0} \cdot(1+a) \text { where } a=u+i v
$$

A combination of $u$ and $v$ is termed a mode. The perturbed term is $(u+i \cdot v)$, and the equivalence between mode amplitude (u) and mode phase (v) is evident. If we now suppose that the (complex) amplitude, $a_{o}$, is the amplitude of a sinusoidal ripple of period $p$, and if the beam travels through one material, it can be show ${ }^{2}$ that $a_{0}$ is transformed by a simple real-element 2 by 2 matrix :

$$
a=\{M\} \cdot a_{0}, \text { or: } \begin{aligned}
& u=m_{11} \cdot u_{0}+m_{12} \cdot v_{0} \\
& v=m_{21} \cdot u_{0}+m_{22} \cdot v_{0}
\end{aligned}
$$

The matrix elements, $m_{11}$ to $m_{22}$, depend only on $\left(p_{1} / p_{c}\right)$ and $B$, defined in (5) and (7). If $p$ is smaller than $\mathrm{p}_{\mathrm{c}}$, the matrix elements are circular sine and cosine of parameters depending on $\mathrm{B}$, and hence produce small gains. If $p$ is larger than $p_{c}$, the matrix elements are hyperbolic sine and cosine, and hence can produce large gain, of the order of $\mathrm{e}^{\mathrm{B}}$ in amplitude, or $\mathrm{e}^{2 \mathrm{~B}}$ in power. This will be illustrated in section 5 . If the beam travels through alternating materials (such as air/glass), the global transformation matrix is, as expected, the product of the individual matrices of each material.

\section{STUDY OF ONE AMPLIFIER SECTION}

With the basic equations we have studied so far, we will now attempt to understand the behavior of one amplifier section, in terms of specification of optics. Because we are studying wavefront error, the input into the model will be a pure $\mathrm{v}$ vector, in which $\mathrm{u}$ (amplitude modulation) is zero. This is a reasonable assumption to first order, and greatly simplifies the mathematics, allowing more insight than retaining all 4 matrix elements.

The output of the model is threefold:

a. We want to limit diffraction loss. This is characterized by the total mode power, $\left(\mathrm{u}^{2}\right.$ $\left.+v^{2}\right)$. 
b. We want to keep power modulation down, to limit damage to the optics. This is characterized by the amplitude part of the vector, $u$.

c. We want to limit wavefront distortion in the lower spatial frequencies; e.g. those that get through the filtering holes (see $\S 3$ ). This is characterized by the phase part of the vector, $v$.

Figure 3 shows the gain curves for the three functions $u^{2}+v^{2}$ (labeled G-vP), $u$ (labeled G-vU) and $v$ (labeled $\mathrm{G}-\mathrm{vV}$ ). The $\mathrm{x}$ axis is ripple size $\mathrm{p}$ and the $\mathrm{y}$ axis is power gain (both log scale). As suggested above $(\S 4.2)$, the gain falls to low values when p becomes smaller than $p_{c}(0.43 \mathrm{~mm})$.

Five regions of interest have been marked off on the graph ${ }^{3}$ (for convenience, values have been rounded a bit):

A. Ripples larger than $33 \mathrm{~mm}$ : diffraction is narrow, so these errors get through the filtering holes, and also fall on the target (angular radius $35 \mu \mathrm{rad}$ approx., equivalent to $33 \mathrm{~mm}$ ripple). The gain function G$\mathrm{vV}$ is almost unity: it is as if non-linearity were zero. Also, Fresnel lengths of such ripples are longer than 1000 meters, so we are entitled to use geometrical optics (wavefront slope, etc.) in this region.

B. Ripples between 33 and $10 \mathrm{~mm}$ : diffraction is narrow, so they get through the filtering holes, but miss the target. The gain function $\mathrm{G}-\mathrm{vV}$ is less than 2 , again almost unity. Fresnel length is between 100 and 1000 meters, almost identical to the laser length. Because of this, it is uncertain whether diffraction or geometric theory should be used ${ }^{3}$.

C. Ripples between 10 and $1 \mathrm{~mm}$ : diffraction is larger than the filtering holes we've chosen for this model, so they cannot add through successive amplifier sections. The gain functions suddenly increase to large values (more than 50), in a smooth way. We are concerned here with the two functions G-vP and G$\mathrm{vU}$ only, since G-vV related to wavefront distortion which does not get out of this amplifier section. In this region, it is to be expected that high loss and damage can occur.

D. Ripples between $I$ and $0.1 \mathrm{~mm}$ : the only difference with case $\mathrm{C}$ is the fact that the gain functions become apparently "chaotic". This is because Fresnel lengths for these ripples rapidly fall below the 1 meter mark, the approximate distance between two consecutive slabs. Thus, maximum gain parameters are decorrelated between two consecutive slabs. We are still concerned with functions G-vP and G-vU, but the average value of G-vP (total power gain) becomes smaller, while peak values of G-vU remain at the same heights.

E. Ripples smaller than $0.1 \mathrm{~mm}$ : gain functions have fallen to almost one, so this region can be treated as conventional scattering loss.

\section{SPECIFICATIONS}

In view of the behavior of laser systems as discussed in chapter 5 above, it seems obvious that the most appropriate language for specifying the source terms to the SRA process, is that of Fourier analysis. For this reason we are investigating specifying the transmitted wavefront in the frequency ranges of $C$ and $D$, and possibly B, of each of the optics in the NIF and LMJ lasers in terms of power spectral density (PSD) and root mean square error (RMS), over the various frequency bands. We won't go over the properties of the PSD and RMS, as they are well known and described elsewhere ${ }^{4}$, but will show how these can be used in ICF optics specifications.

In writing the first revision of the specifications, we chose to define those specifications based on currently available optics. Thus, in this section we will define a specification to obtain the same quality, in terms of the PSD, based on an existing PSD measurement. While this may sound like a specious exercise, it actually is not. The prototype optics used in the Beamlet demonstration project, with a few exceptions already mentioned, are believed to be acceptable for the full-scale production. In section 7, we will show the results obtained using essentially the same approach, to define the specification on a finished amplifier 


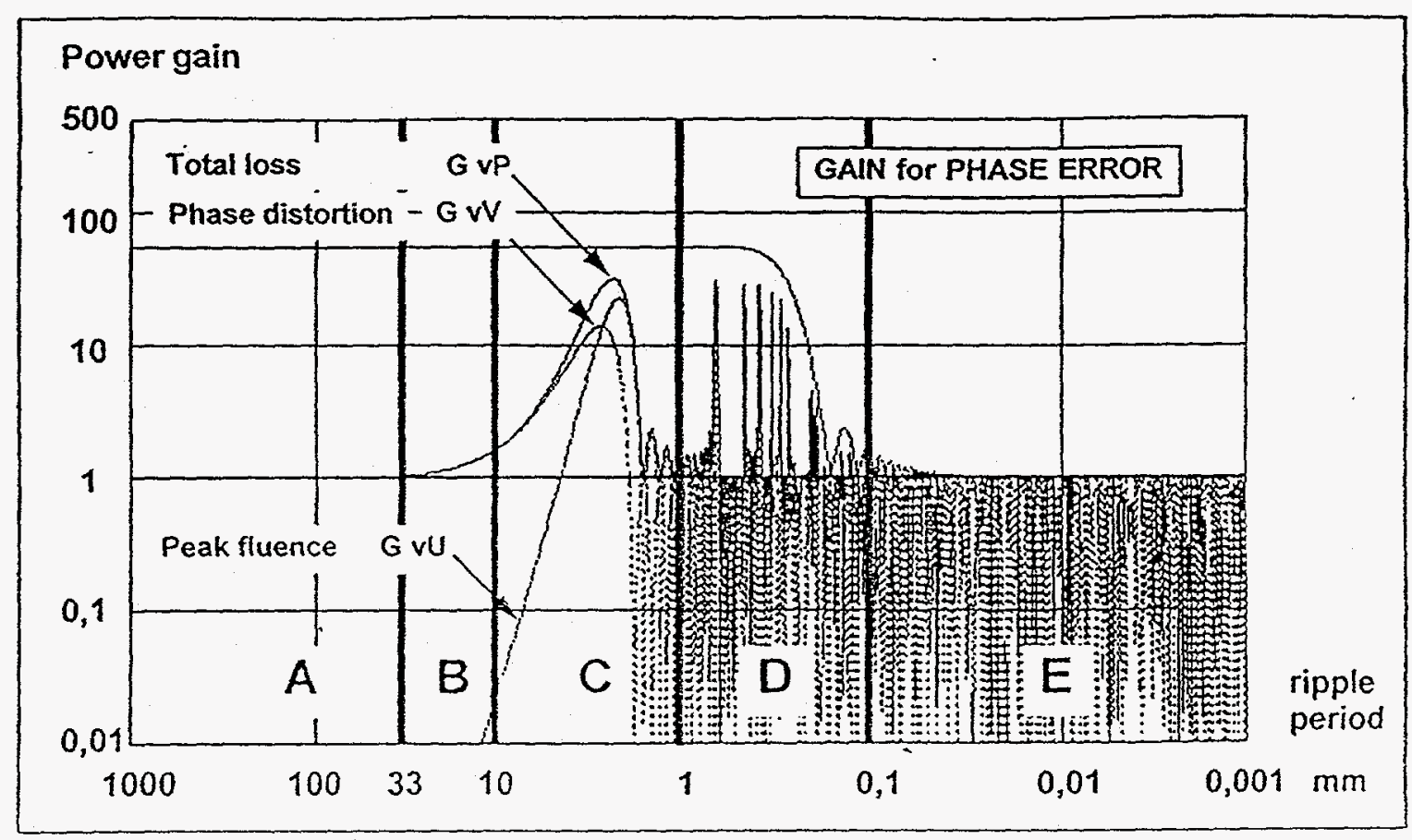

Fig. 3. Gain curves for a typical amplifier section ( 10 slabs, $B=2.0$ ).

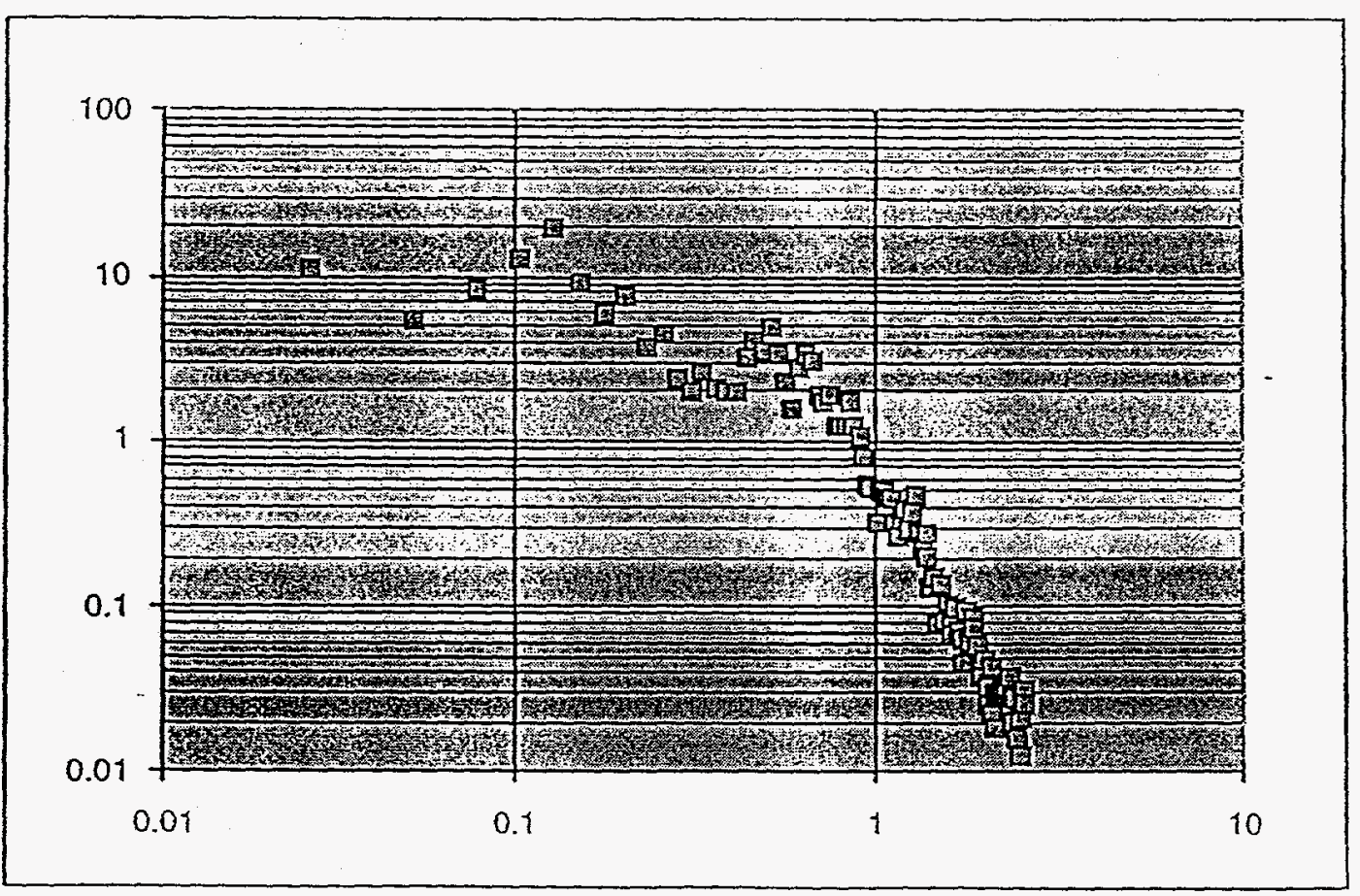

Figure 4. Example of a spatially averaged PSD 
slab. The technology to be used to fabricate the optics for the larger laser system, must be substantially lower in recurring costs than the technology used to fabricate the Beamlet optics. Such a change in technology can be expected to change the PSD of the resultant parts. Thus it is important at this juncture to define a specification which will assure us of 'same quality' optics, to be used by the vendors in qualifying their new processes. The next step may be to begin loosening or tightening this specification based on further modeling or experimentation, or both.

\subsection{Specifying a PSD versus spatial frequency 5}

In figure 4, we see a $\log$-log plot of PSD versus spatial frequency. If we use a straight line to bound the values on this PSD, we effectively place a limit on the amplitude of modulations over a range of frequencies. The slope of the line which bounds the PSD will be determined by the process which created the optic. In the case shown in figure 4, the slope of such a bounding function is about -1 . This means that the specification on the PSD is dropping at a rate of one decade per decade of frequency. Obviously such a bounding function need not be continuous; it may make sense to have lines of different slopes over different regions of the frequency domain.

Such a specification can be shown graphically or analytically, or both. For the example shown in figure 4, a bounding curve could be written as:

$$
\begin{aligned}
& \text { PSD } \leq \text { A } v^{-b} \text { for } 0.08 \mathrm{~mm}^{-1} \leq v \leq 0.63 \mathrm{~mm}^{-1} \\
& \text { where } A=3 \\
& b=1
\end{aligned}
$$

\subsection{Caveats with a PSD specification}

Several other parameters need to be described explicitly in the notation or understood through some other specification being applied ${ }^{6}$. By way of summary, in no particular order, the following parameters have been assumed in writing the above notation as a specification:

1.) number of cycles required per measurement and number of data points per cycle.

2.) specifications regarding scan length for each measurement $(100 \mathrm{~mm}, 7.5 \mathrm{~mm})$.

3.) units of Fourier amplitude (nm).

4.) units of the spatial frequency $(\mathrm{mm}-1)$.

5.) Fourier transform routine to be employed.

6.) I-D or 2-D calculation of the PSD.

7.) number of line-outs averaged to obtain the PSD.

8.) number of phase screens measured against this PSD requirement.

9.) orientation of the line-outs.

10.) the metrology tool to be used or the transfer function of the instrument versus frequency. 7

\subsection{Specifying RMS wavefront error versus frequency}

The above specification controls only the highly periodic functions present on the optic. If we were to use it alone, we could conceivably get the maximum PSD value at all points in the mid-spatial frequency spectrum, which is not our intent. The other key parameter for this specification is the area beneath the PSD curve which, as is described elsewhere ${ }^{4}$, is equivalent to the square of the RMS wavefront error. It is necessary to describe such an RMS requirement in terms of specific frequency ranges. For instance:

$$
\text { RMS } \leq 1.6 \mathrm{~nm} \text { for } 0.08 \mathrm{~mm}^{-1} \leq \mathrm{v} \leq 0.63 \mathrm{~mm}^{-1}
$$

Since one can simply sum the values of the discreet PSD times the frequency increment and take the square root to determine the RMS error, an RMS wavefront requirement translates directly into an equivalent area beneath the curve of the PSD. One could draw another line, the square root of the area beneath which would be indicative of the RMS error, given in nm. The RMS error, in turn, can be converted into total 
scattered energy. By combining the gain curve with the power spectrum, one can calculate the amount of energy which is being transferred to the various spatial frequency regimes for a high energy laser.

Figure 5 shows both a peak PSD and an RMS specification versus frequency in graphical form. Notice that we have drawn the line bounding the top of the area of the RMS specification to be parallel to the peak PSD line from section 6.1. As mentioned before, such a pictorial representation is sufficient; however adding the explicit notation shown in (11) and (12) is more precise, and avoids confusion.

\section{SAMPLE RIPPLE SPECIFICATION}

Thus we are prepared to write a specification for mid-spatial frequency errors in the transmitted wavefront to achieve a given PSD and RMS wavefront error. An example of such a specification is shown graphically in figure 6 . A text section accompanying that figure could be as follows:

Transmitted Wavefront (TWF). Finished Finished amplifier slab transmitted wavefront when measured at use (Brewster's) angle, shall be within the following PSD requirement, when measured and calculated per LLNL (or CEL-V) manufacturing and testing procedures. At least 3 cycles per measurement are required, as well as at least 8 samples per cycle. When plotted log-log, for spatial periods from $33 \mathrm{~mm}$ to $0.12 \mathrm{~mm}$, the maximum value of the PSD shall be less than the line described by the equation:

$$
\begin{aligned}
& \text { PSD } \leq \mathrm{A},-\mathrm{b} \text { for } 0.03 \mathrm{~mm}-1 \leq v \leq 8.5 \mathrm{~mm}-1 \\
& \text { where } \quad \begin{array}{l}
A=1.05 \\
b=1.55
\end{array}
\end{aligned}
$$

as shown graphically in figure 6. The RMS wavefront error, when calculated from the PSD over the spatial period range from $33 \mathrm{~mm}$ to $2.5 \mathrm{~mm}$ (e.g. RMS ${ }_{1}$ ), will be less than $1.8 \mathrm{~nm}$. When calculated over the spatial period range from $2.5 \mathrm{~mm}$ to $0.12 \mathrm{~mm}$ (e.g. RMS2), the RMS wavefront error will be less than $1.1 \mathrm{~nm}$. For demonstration, these RMS values are shown on figure 8 as the square root of the area beneath indicated lines.

In addition, for scale lengths greater than $33 \mathrm{~mm}$, the peak-to-valley $(\mathrm{P}-\mathrm{V})$ transmitted wavefront, measured at use angle, shall be less than $\lambda / 6$, with wavefront gradients less than $\lambda / 30 / \mathrm{cm}$. For scale lengths less than $0.25 \mathrm{~mm}$ the surface roughness shall be less than $0.3 \mathrm{~nm}$, RMS.

\section{CONCLUSION}

We have shown a simple method for generating a PSD specification to control mid-spatial frequency errors on a transmitted wavefront. Power Spectral Density (PSD) has been around for many years now, and is even part of a draft ISO standard on roughness of optical components surfaces ${ }^{8}$. But its usefulness is heightened in systems such as ICF lasers, in which the complicated spatial frequency behavior cannot be mastered with just a few figures. Obviously, the same method can be used to specify any surface or phase function, such as reflected wavefront, as well. The PSD function is useful in understanding the properties of an optical element, especially with respect to high energy lasers. There is no magic to the calculation of a PSD, however care must be taken to properly average and normalize the data correctly. Indeed such calculations are automatic on most modern interferometric systems, however there are some caveats, noted in section 6.2. No simple specification, e.g. peak to valley, gradient, or RMS wavefront, can accurately control the mid-spatial frequency errors, common to large optics made using deterministic processes. The derogatory effects such wavefront errors have on high energy laser systems has been generally addressed. The use of PSD's for calculation of laser performance, and the verification of an optic versus a PSD requirement, are subjects for future talks. 


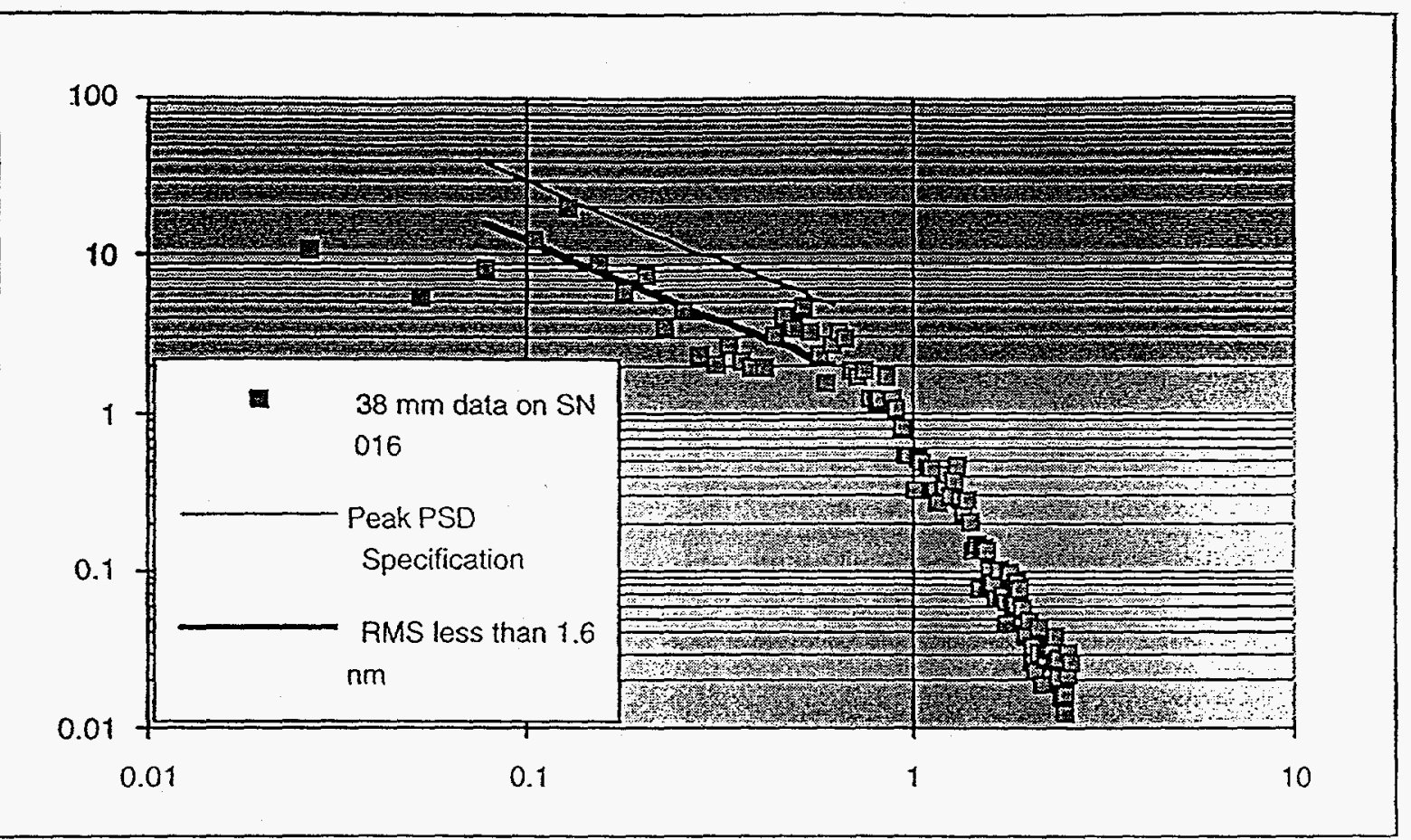

Figure 5. A graphical specification for the Peak PSD over the data valid region of 0.08 $\mathrm{mm}-1$ to $0.63 \mathrm{~mm}-1$. The thin upper line shows the maximum allowable (10 line average) PSD, while the solid line below it is a PSD whose RMS over the frequency domain shown is less than the $1.6 \mathrm{~nm}$ required.

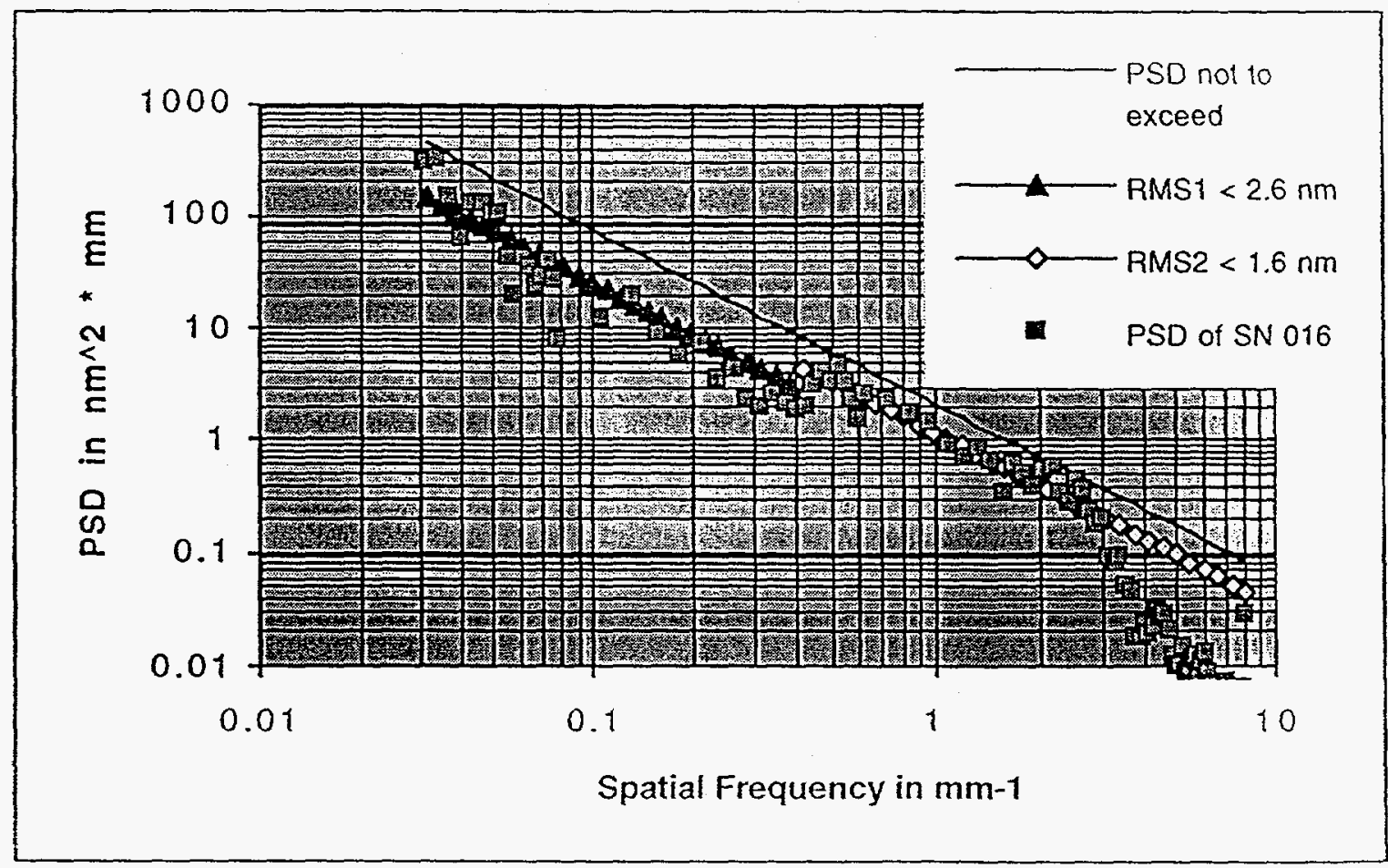

Figure 6. A graphical representation of the transmitted wavefront mid-spatial frequency specification for one of the NIF optics. The specification is to be verified using two measurements, each with 512 pixels per line, and a 10 line spatial average. 


\section{ACKNOWLEDGMENTS}

This work has been conducted in support of optical component development efforts in the ICF Program at the Lawrence Livermore National Laboratory and the development of Laser Méga-Joule (LMJ) at the Centre d'Études de Limeil-Valenton (CEL-V). The authors would like to thank the many individuals involved in this effort at both laboratories, including C. Sauteret, C. Gouedard, K. Manes, J. Lawson, and R. Wolfe, and the members of the Laser Physics Department of CEL-V and Optics Specifications Working Group at LLNL. The work at Livermore was performed under auspices of the US Department of Energy by the Lawrence Livermore National Laboratory under contract No. W-7405-ENG-48. The work at Limeil was performed under the auspices of CEA-DAM, by the Centre d'Études de Limeil-Valenton.

\section{REFERENCES}

1. J.T. Hunt, K.R. Manes, and P.A. Renard, "Hot images from obscurations", Applied Optics Vol. 32, No. 30, pp 5973-5982, October 1993.

2. J.B. Trenholme, "Theory of Irregularity Growth on Laser Beams", 1975 Laser Program Annual Report, Rep. UCRL-50021-75 (LLNL), pp. 237-242.

3. See also C.R. Wolfe and J.M. Auerbach, "Advanced Wavefront Measurement and Analysis for Laser System Modeling", Presentation to Imaging Sciences Workshop at LLNL, November 1994.

4. See, for example, J. M. Bennett and L. Mattsson; "Scattering Theories and Surface Statistics", Chapter 4 in Introduction to Surface Roughness and Scattering, published by OSA: ISBN 1-55752-108-5, 1989.

5. D.M. Aikens, C.R. Wolfe, and J.K. Lawson, "The use of PSD functions in specifying optics for the National Ignition Facility", in Optical Fabrication and Testing, Proc. SPIE 2576, June 1995.

6. J.M. Elson and J.M. Bennett, "Calculation of the power spectral density from surface profile data", Applied Optics, Vol 34, No. 1, p. 201 (1995).

7. E. L. Church et al; "Direct comparison of mechanical and optical measurements of the finish of precision machined optical surfaces", Opt. Eng., Vol. 24, No. 3, May/June 1985.

8. "Optics and optical instruments-Preparation of drawings for optical elements and systems, Part 8: Surface texture", ISO/DIS 10110-8, March 1994. 


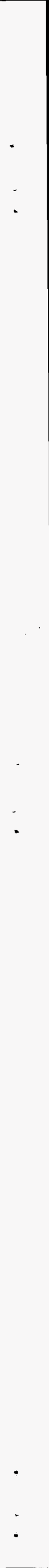

